\title{
Stimulus nonequivalences in go/no-go avoidance discriminations: Sensory, drive, and response factors*
}

\author{
MARINA FRONTALI \\ Istituto di Psicologia del Consiglio Nazionale delle Ricerche \\ Via dei Monti Tiburtini, 509, Roma, Italy \\ and \\ GIORGIO BIGNAMI \\ Psychopharmacology Section, Department of Therapeutic Chemistry \\ Istituto Superiore di Sanità, Roma, Italy
}

\begin{abstract}
Previous work with rats showed slower active avoidance (AA) and passive avoidance (PA) learning in a light-go/noise-light no-go task $\left(\mathrm{L}^{+} \mathrm{NL}^{-}\right)$than in a noise-go/light-noise no-go task $\left(\mathrm{N}^{+} \mathrm{LN}^{-}\right)$, independently of noise intensity and of presence or absence of AA pretraining. Experiment I compared these two tasks and the corresponding discriminations with a compound AA and a simple PA signal. The latter were learned at a faster rate, with only negligible differences between the two types of stimulus arrangements $\left(\mathrm{NL}^{+} \mathrm{L}^{-}\right.$vs $\left.\mathrm{LN}^{+} \mathrm{N}^{-}\right)$. Experiment II investigated the acquisition and reversal of four go/no-go tasks with a simple AA signal, with either a simple or a compound no-go signal and asymmetrical reinforcement, i.e., with an extinction instead of a PA contingency during the presentation of no-go signals. As in previous experiments with symmetrical reinforcement, the groups with a simple no-go signal $\left(\mathrm{L}^{+} \mathrm{N}^{-}, \mathrm{N}^{+} \mathrm{L}^{-}\right)$ acquired AA with little or no generalization to the other stimulus. In reversal, however, the $\mathrm{L}^{+} \mathrm{N}^{-}$group (formerly $\mathrm{N}^{+} \mathrm{L}^{-}$) responded to the no-go signal much more frequently than the other $\left(\mathrm{N}^{+} \mathrm{L}^{-}\right.$, formerly $\mathrm{L}^{+} \mathrm{N}^{-}$). Both the $\mathrm{L}^{+} \mathrm{NL}^{-}$and the $\mathrm{N}^{+} \mathrm{LN}^{-}$groups gave a large number of responses to the extinction signal, but the differences between the two groups were at least as large as in previous work with a PA contingency. These data show that perceptual or attentional phenomena cannot explain stimulus nonequivalences in situations with a compound no-go signal. Furthermore, the similar nonequivalences found in situations with and without differential fear conditioning (AA-extinction tests vs AA-PA tests) leads to complex, highly speculative models concerning possible interactions between response and drive-modulating properties of various stimuli.
\end{abstract}

The problem of nonequivalence of stimuli in the acquisition and performance of various types of conditioned responses has recently received renewed attention (Seligman, 1970; Shettleworth, 1972). As concerns situations with negative reinforcement, it is well known that visual and acoustic stimuli readily acquire aversive properties in rats when paired with discrete punishing events such as electric shocks or air blasts, but not when an "illness"-inducing agent is used as the US, while the opposite applies to gustatory and olfactory stimuli (Revusky \& Garcia, 1970). Furthermore, stimulus factors have of ten been shown to play an important role in the acquisition of active avoidance responses. However, while it is well known that buzzer is a more efficient avoidance CS than light (Smith, McFarland, \& Taylor, 1961; Biederman, 1967), it appears that stimulus modality per se cannot account for all the differences observed (see, e.g., the experiments comparing buzzer, white noise, tone, and light CSs reported by Myers, 1964).

It is well known that stimulus factors must be considered in connection with other factors, particularly response factors. In fact, it appears that inbuilt (species-specific) stimulus-response relationships or

*We wish to acknowledge the assistance of Luigi De Acetis and Luigi Amorico in running the animals and in preliminary data analyses. hierarchies can favor or hinder the conditioning of a given response to a given stimulus, either in general or with in a given US category (Konorski, 1967; Shettleworth, 1972). Furthermore, there are data which show that interactions between stimuli of the same or different modalities can be more important than main effects of stimulus differences, for example, the work on startle responses by Ison and Hammond (1971).

As concerns avoidance situations, a striking instance of stimulus nonequivalence was observed in experiments on successive discrimination learning by rats using an active avoidance (AA) contingency upon presentation of a given signal (go signal, $\mathrm{CS}^{\dagger}$ ) and a passive avoidance (PA) contingency upon presentation of a stimulus complex including the $\mathrm{CS}^{+}$and a stimulus of a different modality (no-go stimulus complex, $\mathrm{CS}^{-}$) (Rosic, Frontali, \& Bignami, 1969; Rosic̀ \& Bignami, 1970; Frontali \& Bignami, 1973). In fact, a noise-go/light-noise no-go task $\left(\mathrm{N}^{+} \mathrm{LN}^{-}\right)$was learned much faster than a light-go/noise-light no-go task ( $\left.\mathrm{L}^{+} \mathrm{NL}^{-}\right)$. Part of the difference was due, as expected, to the higher efficiency of noise than of light as AA signal, but the $\mathrm{L}^{+} \mathrm{NL}^{-}$also showed a marked impairment of PA. The latter finding could not be ascribed to the use of a compound $\mathrm{CS}^{-}$ per se, nor to differences in the acquisition of response-suppressing properties by visual and acoustic stimuli per se, since other groups of animals learned the 
$\mathrm{N}^{+} \mathrm{L}^{-}$and $\mathrm{L}^{+} \mathrm{N}^{-}$tasks at a similar rate. In summary, the efficiency of a compound visual and acoustic signal as PA signal seemed to depend on which of the two components was used as AA signal. Furthermore, such interaction was little affected by the type of the acoustic signal used (buzzer vs speech noise), by the intensity of the acoustic signal itself (speech noise of $90 \mathrm{~dB}$ vs speech noise of $70 \mathrm{~dB}$ ), and by the presence or absence of $\mathrm{AA}$ training prior to discrimination training.

Considering that noise elicits more running and jumping than light, the differences in PA learning were tentatively explained as follows. The $\mathrm{L}^{+} \mathrm{NL}^{-}$task was viewed as a situation in which a stimulus that tends to elicit unconditioned running responses $(\mathrm{N})$ is unsuitable to suppress conditioned locomotor responses elicited by an AA signal (L). The $\mathrm{N}^{+} \mathrm{LN}^{-}$task, on the contrary, was viewed as providing a signal that can easily trigger "behavior other than running" (L), so as to suppress punished running to another signal with both unconditioned and conditioned motorigenic properties (N).

The available data, however, did not entirely exclude alternative explanations of the above interactions. As concerns perceptual and attentional processes, it appeared possible that masking or overshadowing of noise by light could lead to a greater difficulty of the $\mathrm{L}^{+} \mathrm{NL}^{-}$discrimination. This hypothesis, predicting that not only discriminations with simple go signals and compound no-go signals but also discriminations with opposite response requirements $\left(\mathrm{NL}^{+} \mathrm{L}^{-}\right.$and $\left.\mathrm{LN}^{+} \mathrm{N}\right)$ would be learned at different rates, was tested in Experiment I.

\section{EXPERIMENT I}

\footnotetext{
Method

Materials and methods were similar to those of a previous experiment (Frontali \& Bignami, 1973). Ss were 64 adult male albino rats of a Wistar-derived strain. The apparatus consisted of a series of $490 \times 270 \times 225 \mathrm{~cm}$ shuttleboxes without central partition or barrier, placed inside cork-lined wooden containers providing sound insulation. A nondirectional light stimulus was provided by two $10-\mathrm{W}$ bulbs mounted at the center of the ceiling. A nondirectional acoustic stimulus (speech noise) was provided by a Grason-Stadler generator (Model 901 B) connected to a 4-in. loudspeaker located behind the shuttlebox in a central position. The generator was set so as to obtain a 90-dB intensity inside the shuttlebox, as measured by a Brüel and Kjaer sound-level meter (Type 2203) placed at floor level. Scrambled shock of $1.5 \mathrm{~mA}(105 \mathrm{~V}$ with a $70 \mathrm{~K}$-ohm resistance in series) was given through the grid floor.

The animals were trained for 18 sessions (Monday through Saturday for 3 consecutive weeks). Each session consisted of 50 trials at $30-\mathrm{sec}$ intervals. Twenty-five AA and 25 $P A$ trials were given according to the sequence (AA-PA-AA-PA-PA-AA-PA-AA; AA ...) repeated six times, plus one $A A$ and one PA trial. A crossing response during the CS-US interval (see below) in an AA trial terminated the CS and prevented shock administration, while a response after shock onset terminated both CS and US at the same time. Passive avoidance signals (see below) had a fixed duration. Both respor ses during these signals and responses during the intertrial interval (ITI) were punished by shock until the animal returned to the opposite side of the box.
}

The animals were subdivided at random in to four groups of 16 rats each. Two groups received simple AA and compound PA stimuli, while the other two received compound AA and simple PA stimuli. Simple stimuli were either light (L) or noise (N), while compounds consisted of a stimulus identical to the one presented alone, preceded by $1 \mathrm{sec}$ and accompanied for its whole duration by the stimulus of the other modality. This led to a 2 by 2 factorial design, one factor being the contingency (AA or PA) associated with the simple stimulus ("simple stimulus contingency," $\mathrm{L}^{+} \mathrm{NL}^{-}$and $\mathrm{N}^{+} \mathrm{LN}^{-}$vs $\mathrm{NL}^{+} \mathrm{L}^{-}$and $\mathrm{LN}^{+} \mathrm{N}$ ) and the other being the modality of the stimulus presented alone ("simple stimulus modality," $\mathrm{L}^{+} \mathrm{NL}^{-}$and $\mathrm{NL}^{+} \mathrm{L}^{-}$vs $\mathrm{N}^{+} \mathrm{LN}^{-}$and $\mathrm{LN}^{+} \mathrm{N}^{-}$). As in previous experiments, the CS-US interval was $5 \mathrm{sec}$ in AA trials when the AA stimulus was a simple one, while discriminations with a compound AA signal used an interval of $5 \mathrm{sec}$ between the onset of the second component and the US, i.e., an interval of $6 \mathrm{sec}$ between the onset of the first component and the US. Simple PA signals lasted $6 \mathrm{sec}$. As in previous experiments, compound PA signals consisted of a 6 - and a 5 -sec stimulus which overlapped, had a fixed duration, and terminated simultaneously.

\section{Results}

The data on total errors presented in Fig. 1 show that discriminations with compound AA stimuli were learned faster than discriminations with compound PA stimuli. This difference was more marked in the first 2 weeks of training and produced a significant interaction between simple stimulus contingency and sessions in a 2 by 2 analysis of variance with repeated measures (Winer, 1962) $(\mathrm{F}=4.01, \quad \mathrm{df}=17 / 1020, \mathrm{p}<.001)$. As in previously reported experiments, the $\mathrm{N}^{+} \mathrm{LN}^{-}$group performed better than the $\mathrm{L}^{+} \mathrm{NL}^{-}$group, while a slight superiority of the $\mathrm{LN}^{+} \mathrm{N}^{-}$group appeared only at the end of training. In fact, the analysis of variance showed both a two-way interaction of simple stimulus modality and sessions $(F=1.79, d f=17 / 1020, p<.05)$ and a three-way interaction between the two stimulus factors and sessions $(F=2.52, \mathrm{df}=17 / 1020, \mathrm{p}<.005)$.

The data on omission and commission errors (Fig. 1) indicate that the groups with compound AA signals were superior to the others both in AA learning and in PA learning. The analysis of variance on omission errors showed a significant interaction between simple stimulus contingency and sessions, probably due to the variable size of the differences from one session to another $(F=3.89, \mathrm{df}=17 / 1020, \mathrm{p}<.001)$. On the other hand, a main effect of simple stimulus contingency was found in the analysis on commission errors $(F=7.49, \mathrm{df}=1 / 60$, $\mathrm{p}<.01)$. As in previous experiments, the $\mathrm{N}^{+} \mathrm{LN}^{-}$was superior to the $\mathrm{L}^{+} \mathrm{NL}^{-}$group in $\mathrm{AA}$, the difference being larger in the central portion of the training period. On the other hand, little difference in AA was observed between the $\mathrm{LN}^{+} \mathrm{N}^{-}$and the $\mathrm{NL}^{+} \mathrm{L}^{-}$groups. In fact, a three-way interaction between the two stimulus factors and sessions was found in the analysis on omission errors $(F=3.02, \quad d f=17 / 1020, \quad p<.001)$. The higher frequency of commission errors in the $\mathrm{L}^{+} \mathrm{NL}^{-}$than in the $\mathrm{N}^{+} \mathrm{LN}^{-}$group was paralleled by a similar, although much smaller, difference between the $\mathrm{NL}^{+} \mathrm{L}^{-}$and the $\mathrm{LN}^{+} \mathrm{N}^{-}$ groups. This allowed the appearance of a significant 
main effect of simple stimulus modality in the analysis of commission errors $(\mathrm{F}=10.08, \mathrm{df}=1 / 60, \mathrm{p}<.005)$.

\section{Discussion}

The experiment reported above showed similar rates of learning of the two discriminations with compound $\mathrm{AA}$ and simple PA stimuli $\left(\mathrm{NL}^{+} \mathrm{L}^{-}\right.$and $\left.\mathrm{LN}^{+} \mathrm{N}^{-}\right)$. Therefore, it appears unlikely that perceptual or attentional factors perse are responsible for the differences repeatedly found between tasks with simple $\mathrm{AA}$ and compound PA stimuli $\left(\mathrm{L}^{+} \mathrm{NL}^{-}\right.$and $\left.\mathrm{N}^{+} \mathrm{LN}\right)$. This is in agreement with previous data showing that the differences between the $\mathrm{L}^{+} \mathrm{NL}^{-}$and $\mathrm{N}^{+} \mathrm{LN}^{-}$tasks are maintained at widely differing intensities of the noise stimulus (Frontali \& Bignami, 1973), while it is well known that overshadowing phenomena are strongly affected by stimulus intensity (e.g., Mackintosh, 1971).

An analysis of stimulus nonequivalences must also consider that the groups trained with compound AA stimuli showed an overall better performance than did the groups with compound PA signals. On the one hand, faster AA learning with a compound go signal could be ascribed to the use of a slightly longer CS-US interval ( $6 \mathrm{sec}$ from the onset of the first presented stimulus to the onset of shock instead of $5 \mathrm{sec}$ ) and/or to a stimulus intensity dynamic effect, assuming that the physical energy of a compound is higher than that of any one of the components. On the other hand, a unitary explanation for the differences in both $\mathrm{AA}$ and $\mathrm{PA}$ learning can be attempted by using the model of Weiss (1972), which is based on a continuum including all possible on and off states of two stimuli. Although the situations considered here differ in many respects from those on which the Weiss model is based, the $\mathrm{L}^{+} \mathrm{NL}^{-}$and $\mathrm{N}^{+} \mathrm{LN}^{-}$discriminations can be viewed as tasks in which a given response meets reinforcement requirements at the two extremes of the continuum, while an opposite type of response meets reinforcement requirements when an intermediate combination is presented. In fact, behavior other than crossing (PA) is required both in the absence and in the presence of the two stimuli, while crossing (AA) is required either in the light-on/noise-off condition or in the light-off/noise-on condition. Fewer ambiguities are present when the compound stimulus is associated with an AA contingency. In fact, crossing is required at one extreme of the continuum (both stimuli on), while behavior other than crossing is required both with an intermediate combination (light-on/noise-off or light-off/noise-on) and at the opposite extreme of the continuum (both stimuli off).

All data so far obtained strengthen the conclusion that stimulus nonequivalences cannot be explained by various types of sensory or attentional factors but must be explained by taking into account sensory and response factors at the same time. Since different mechanisms may underlie various types of response elimination, the experiment reported below extended the study of stimulus-response interactions to situations

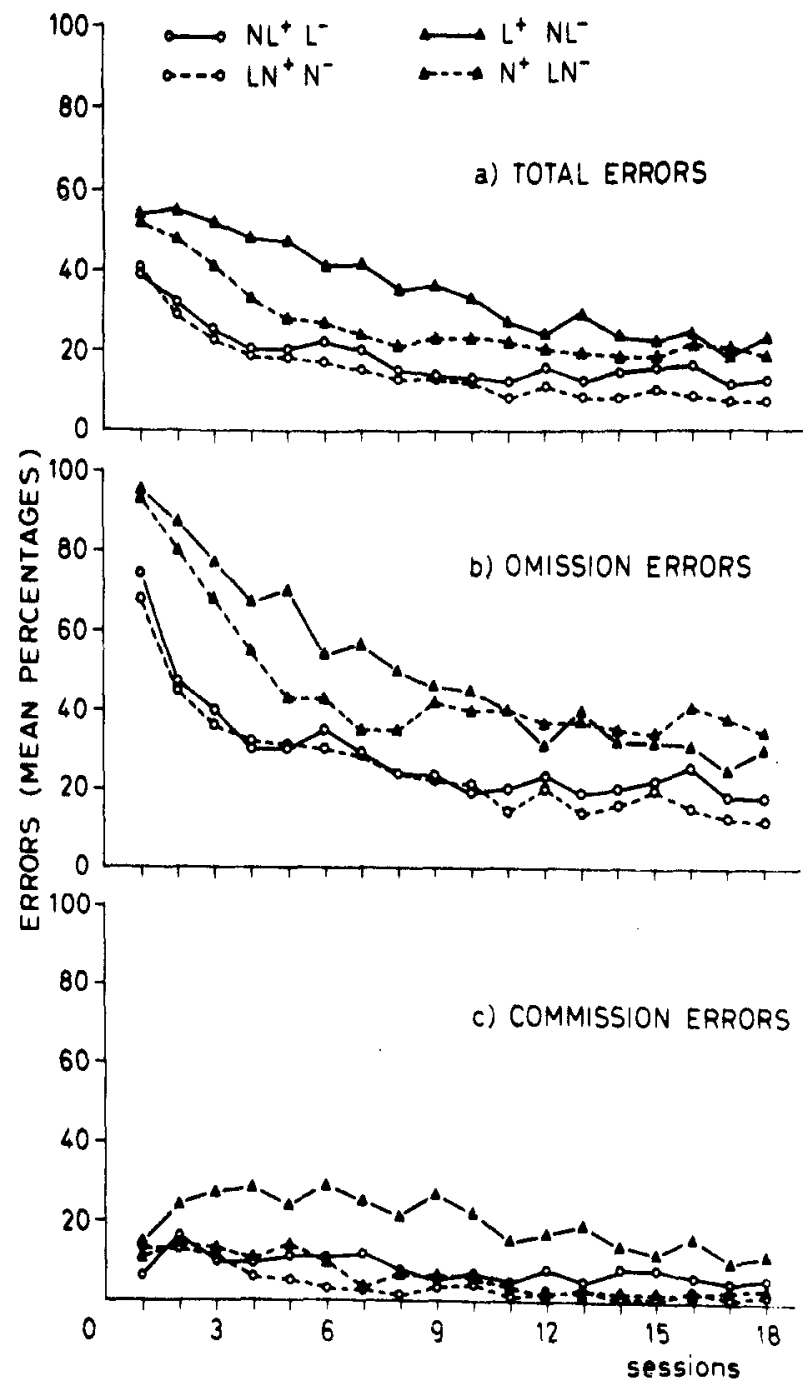

Fig. 1. Discrimination learning in a shuttlebox with light (L) and noise $(\mathrm{N})$ stimuli. Four stimulus arrangements were used, i.e., either simple active avoidance and compound passive avoidance signals ( $\mathrm{L}^{+} \mathrm{NL}^{-}$and $\mathrm{N}^{+} \mathrm{LN}$ ) or vice versa $\left(\mathrm{NL}^{+} \mathrm{L}^{-}\right.$and $L^{+} N$ ). Omission errors are active avoidance failures and commission errors are passive avoidance failures.

with an extinction instead of a PA contingency in no-go trials.

\section{EXPERIMENT II}

\section{Introduction}

The data of Experiment I reported above and those of previous studies indicate that most of the animals receive many shocks during the acquisition of the $\mathrm{L}^{+} \mathrm{NL}^{-}$and the $\mathrm{N}^{+} \mathrm{LN}^{-}$tasks, both for nonresponding in AA trials and for responding in PA trials. Therefore, these discriminations can be viewed as tasks requiring a motor act differentiation control (crossing vs behavior other than crossing) under the same drive operation (all CS paired to the same US) (Konorski, 1967). Incidentally, it must be recalled that such "behavior other than crossing" during successful response withholding in PA 
trials can include widely differing response repertoires, depending on the internal state of the animal. Well trained, untreated animals show rearing and orienting responses during presentation of the PA signal. On the other hand, the same rats can compensate for the deficit caused by a response-disinhibiting drug (scopolamine) by taking short runs within the safe part of a shuttlebox without central partition or barrier and stopping just before entering the electrified part of the box (Carro-Ciampi \& Bignami, 1968, Experiment 2).

At this point, it should be considered that marked differences in stimulus factors have been shown between successive discriminations in which similar drive states are elicited, but different responses are required upon presentation of different stimuli and successive discriminations in which different drive states are elicited by the various stimuli. For example, tones of different frequencies from the same source are used much more efficiently in successive go/no-go discriminations with asymmetrical positive reinforcement than in successive go-left/go-right discriminations with symmetrical positive reinforcement, while the converse is true in the case of identical stimuli from spatially separated sources (Eawicka, 1964; Dobrzecka, Szwejkowska, \& Konorski, 1966). This led Konorski to hypothesize that signaling systems can operate in two basically different ways, the relative importance of which varies from one to the other type of discrimination, thereby underlining different types of preparedness. The first allows drive changes in different directions (e.g., conditioned drive activation vs conditioned drive inhibition) upon exposure to different stimuli, while the second allows response choice, given the same drive operation (for additional examples and a more comprehensive theoretical analysis, see Konorski, 1967).

The implications of this hypothesis are clear when comparing successive discriminations with similar AA contingencies but with different contingencies in no-go trials (PA vs extinction during the no-go signal). In fact, differential responding to the go signal can be achieved through differential fear conditioning in the AA-extinction task (i.e., the instrumental equivalent of Pavlovian conditioned inhibition tests with negative reinforcement) but not in the AA-PA task. The attenuation or elimination of stimulus nonequivalences in the former situation would confirm and extend the Konorski hypothesis by showing different types of preparedness in instrumental discriminations based on classical fear conditioning and extinction, as compared to discriminations requiring two incompatible responses under the same drive operation. On the other hand, the finding of stimulus nonequivalences also in discriminations with asymmetrical reinforcement would lead to more complex, highly tentative models. Since the results of Experiment II were largely in this direction, these models will be discussed later.

The experiment reported below included a study of the effects of cue reversal, allowing a further investigation of two points. First, this procedure can measure possible differences in the inhibitory control by previously nonreinforced stimuli or stimulus complexes on the basis of differential rates of AA reconditioning. Second, it appeared important to assess whether or not there are stimulus nonequivalences in reversal in addition to or other than those found during original discrimination training. In fact, reversal tests may prove or disprove the hypothesis that an absence of stimulus nonequivalence in the $\mathrm{L}^{+} \mathrm{N}^{-}$and $\mathrm{N}^{+} \mathrm{L}^{-}$tasks is due to a "floor effect," namely, to the inconspicuousness of generalization phenomena across simple stimuli of different modalities during acquisition.

Quite obviously, this experimental design implied the elimination of intertrial response (ITR) punishment, besides the elimination of punishment for responding to the no-go signal. In fact, ITR punishment, by exposing the animal to shocks in absence of discrete exteroceptive signals, probably maintains a high level of fear of general apparatus cues. This was clearly undesirable in an experiment designed to investigate the role of stimulus interactions in situations as close as possible to classical differentiation and conditioned inhibition tests.

\section{Method}

Ss were 72 rats of the same strain as that used in Experiment I. The apparatus and stimulus characteristics were the same as in Experiment $I$. The rats were subdivided at random into four groups of 18 animals each according to a 2 by 2 design, one factor being the modality of the stimulus used as AA signal ("go stimulus modality," L vs N) and the other being the use of a simple or a compound no-go stimulus ("no-go stimulus type," $\mathrm{N}$ or $\mathrm{L}$ vs $\mathrm{NL}$ or $\mathrm{LN}$ ). The AA contingencies were the same as for the groups trained with a simple AA stimulus in Experiment I (5-sec CS-US interval, delay procedure). Responses during the no-go stimulus and during ITIs were not punished, while the characteristics of no-go stimuli were the same as in Experiment I (fixed duration of $6 \mathrm{sec}$ in the case of simple stimuli and first presented stimuli in compounds, fixed duration of $5 \mathrm{sec}$ in the case of the shorter stimuli in compounds). After 18 acquisition sessions given as in Experiment $I$, all animals received additional training with reversed stimulus conditions. This included 16 sessions distributed irregularly over a period of 5 weeks, with all groups treated equally as concerns distribution of practice.

\section{Results}

Original Training. The data shown in Fig. 2 indicate that the groups with simple no-go stimuli learned faster than the groups with compound no-go stimuli. These differences, regarding both omission and commission errors, showed opposite trends over time. In fact, as the animals learned to avoid a higher and higher proportion of the scheduled shocks, the differences in AA became attenuated. On the other hand, all groups showed progressively higher rates of responding to no-go signals, and between-group differences reached their maximum only after several days of training. This situation was confirmed by the finding of significant interactions between no-go stimulus type and sessions in the 2 by 2 analyses for repeated measures (total errors: $F=2.65$, 


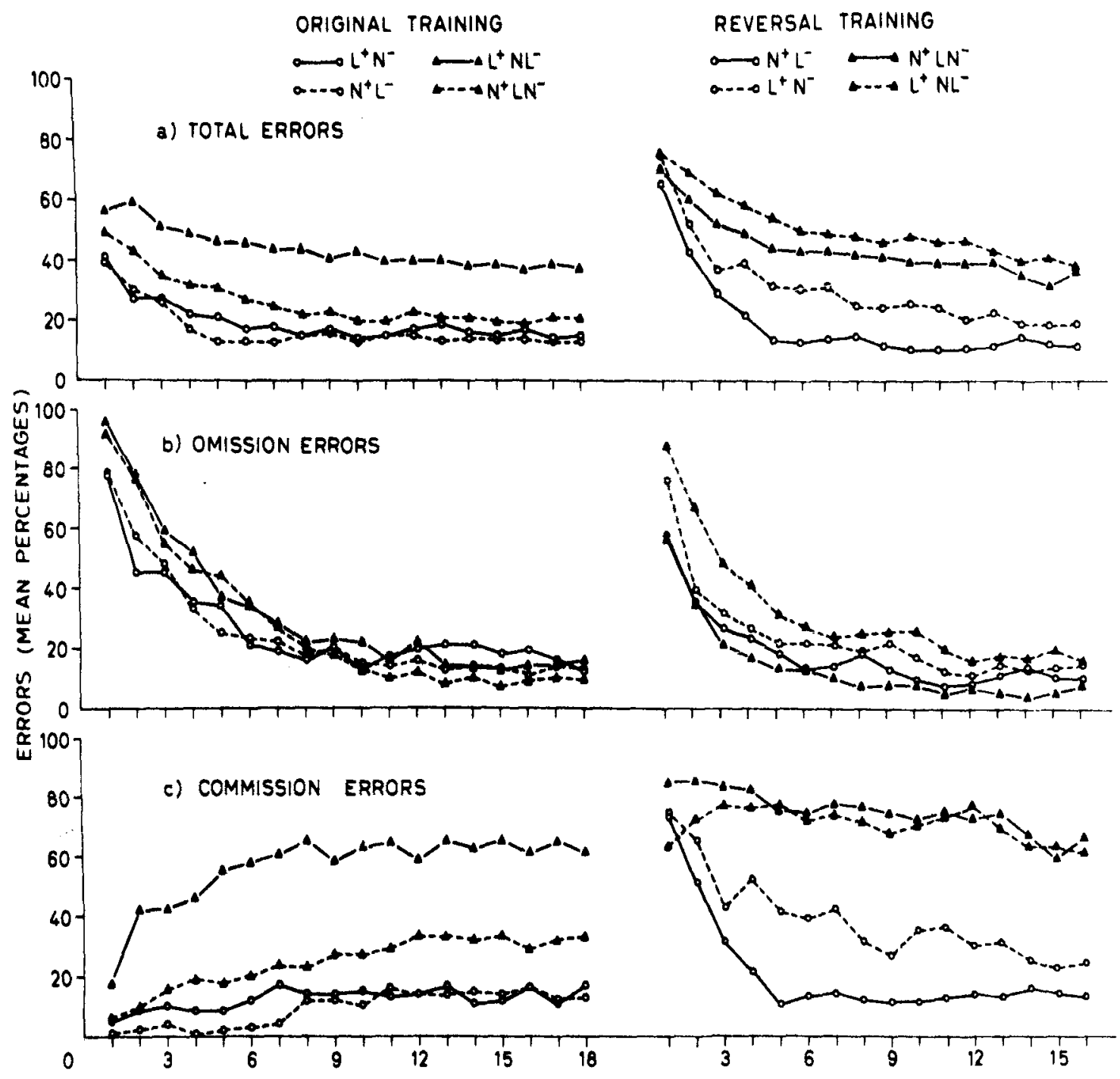

Fig. 2. Discrimination learning and reversal in a shuttlebox.with either light $(L)$ or noise $(N)$ as active avoidance signals and either simple or compound nonreinforced (extinction) signals $\left(\mathrm{L}^{+} \mathrm{N}^{-}\right.$and $\mathrm{N}^{+} \mathrm{L}^{-}$vs $\mathrm{L}^{+} \mathrm{NL}^{-}$and $\left.\mathrm{N}^{+} \mathrm{LN}\right)$. Omission errors are active avoidance failures, while commission errors are failures to withhold responses in nonreinforced trials.

$\mathrm{df}=17 / 1156, \mathrm{p}<.001 ;$ omission errors: $\mathrm{F}=7.46$, $p<.001$; commission errors: $F=3.80, p<.001$ ).

Only negligible differences were observed between the $\mathrm{L}^{+} \mathrm{N}^{-}$and the $\mathrm{N}^{+} \mathrm{L}^{-}$groups, while the $\mathrm{L}^{+} \mathrm{NL}^{-}$animals gave many more responses to the nonreinforced signal than did the $\mathrm{N}^{+} \mathrm{LN}^{-}$rats. In fact, a significant interaction between go stimulus modality and no-go stimulus type was obtained both in the analysis on commission errors $(\mathrm{F}=11.71, \mathrm{df}=1 / 68, \mathrm{p}<.01)$ and in the analysis on total errors $(F=14.80, \mathrm{df}=1 / 68, \mathrm{p}<.001)$. The very high frequency of commission errors in the $\mathrm{L}^{+} \mathrm{NL}^{-}$group, particularly at a time when most animals had reached a high level of AA, was obviously due to the fact that about $80 \%$ of the rats gave little or no evidence of differential responding, while $20 \%$ reached a criterion of $75 \%$ correct in both AA and extinction trials. (Another experiment, using a much more extended training period with irregularly distributed periods of rest, showed that at least $50 \%$ of the $\mathrm{L}^{+} \mathrm{NL}^{-}$rats eventually reach the above criterion. Furthermore, most of the remaining animals eventually respond with a significantly greater frequency to the go than to the no-go signal.)

Representative data on ITR rates at the end of the first, second, and third week of training are reported in Table 1. These data show a somewhat greater frequency of these responses in the $\mathrm{L}^{+} \mathrm{N}^{-}$and $\mathrm{L}^{+} \mathrm{NL}^{-}$groups than in the others, which was confirmed by the finding of a main effect of go stimulus modality in the analysis of variance $(F=4.91, \mathrm{df}=1 / 68, \mathrm{p}<.05)$.

Reversal Training. As in the case of original training, the data on reversal training showed faster discrimination learning with simple than with compound no-go stimuli. Also, in this instance the differences in various error scores showed opposite trends over time. In fact, the differences in omission errors were larger in the first part of the training period, while those regarding commission and total errors were larger after a certain period of training. All analyses showed a significant 
Table 1

Intertrial Response Rates in Experiment II

\begin{tabular}{lccc}
\hline & \multicolumn{3}{c}{ ITRs Per } \\
Group & Session 6 & Session 12 & Session 18 \\
\hline $\mathrm{L}^{+} \mathrm{N}^{-}$ & $1.29 \pm 0.26$ & $1.58 \pm 0.27$ & $1.59 \pm 0.24$ \\
$\mathrm{~N}^{+} \mathrm{L}^{-}$ & $0.65 \pm 0.13$ & $1.20 \pm 0.18$ & $1.18 \pm 0.19$ \\
$\mathrm{~L}^{+} \mathrm{NL}^{-}$ & $1.21 \pm 0.22$ & $1.69 \pm 0.32$ & $1.25 \pm 0.19$ \\
$\mathrm{~N}^{+} \mathrm{LN}^{-}$ & $0.74 \pm 0.25$ & $1.21 \pm 0.25$ & $1.15 \pm 0.19$ \\
\hline
\end{tabular}

interaction between no-go stimulus type and sessions (total errors: $\mathrm{F}=7.93, \mathrm{df}=15 / 1020, \mathrm{p}<.001$; omission errors: $F=2.16, p<.01$; commission errors: $F=11.48$, $\mathrm{p}<.001)$.

On the other hand, the profile of effects due to go stimulus modality was different from that observed during original training. Both groups undergoing reversal training with noise as AA signal made fewer total and omission errors than the corresponding groups with light as AA signal. Presumably due to the initially smaller size of the differences in the case of total errors and to a progressive attenuation of the effect in the case of omission errors, both analyses showed a significant interaction between go stimulus modality and sessions (total errors: $\mathrm{F}=1.73, \mathrm{df}=15 / 1020, \mathrm{p}<.05$; omission errors: $F=2.92, p<.001)$. As concerns commission errors, the group undergoing reversal training with the $\mathrm{L}^{+} \mathrm{N}^{-}$contingencies showed greater resistance to extinction than did the $\mathrm{N}^{+} \mathrm{L}^{-}$group, while the $\mathrm{N}^{+} \mathrm{LN}^{-}$and $\mathrm{L}^{+} \mathrm{NL}^{-}$groups showed similarly high rates of responding to the no-go signal. This explains the finding of a significant interaction between go stimulus modality and no-go stimulus type in the analysis on commission errors $(\mathrm{F}=4.79, \mathrm{df}=1 / 68, \mathrm{p}<.05)$.

ITR data showed initially higher response rates during the reversal than during the initial training period. These data, yielding both an interaction between go stimulus modality and sessions $(\mathrm{F}=1.99, \mathrm{df}=15 / 1020, \mathrm{p}<.05)$ and an interaction between no-go stimulus type and sessions $(F=3.06, d f=15 / 1020, p<.001)$, are too complex to be reported in detail. Suffice it to say that the between-group differences in ITR rates may have contributed to the above differences in AA performance but not to differences in extinction responding. In fact, both the interaction between the two stimulus factors and the three-way interaction remained far from statistical significance.

\section{Discussion}

The results obtained in the original training phase indicate that the acquisition of go/no-go avoidance tasks with asymmetrical reinforcement does not differ substantially from that of corresponding tasks with symmetrical reinforcement, except for an overall greater frequency of responses to the no-go signal. Such increase in commission errors is not surprising, since not only the absence of a PA contingency reduced error cost, but also the overall reduction of shock frequency, partly due to the elimination of ITR punishment, may have attenuated the tendency to freeze. The differences between the $\mathrm{L}^{+} \mathrm{NL}^{-}$and the $\mathrm{N}^{+} \mathrm{LN}^{-}$conditions previously observed in AA-PA tests were not only maintained in AA-extinction tests, but even increased. The similarity of the two groups with simple go and no-go stimuli $\left(\mathrm{L}^{+} \mathrm{N}^{-}\right.$and $\left.\mathrm{N}^{+} \mathrm{L}^{-}\right)$confirms that the above differences were due to interactions between stimuli of different modalities, not to stimulus modality per se. Furthermore, the ITR data exclude that these differences were due to a general enhancement of locomotor activity in the $\mathrm{L}^{+} \mathrm{NL}^{-}$condition as compared to the $\mathrm{N}^{+} \mathrm{LN}^{-}$condition.

The groups trained with simple no-go stimuli not only showed little generalization of responding from reinforced to nonreinforced trials but also acquired the AA response faster than the corresponding groups with compound no-go stimuli. This difference can be explained by considering that the conditioned inhibition paradigm $\left(\mathrm{CS}^{+}\right.$reinforced, $\mathrm{CI} / \mathrm{CS}^{+}$nonreinforced $)$ implied a partial reinforcement of the AA signal. Although a direct quantitative comparison with the experiments of Katzev and Enkema (1973) cannot be made, the latter show that avoidance learning is retarded when the animal is not shocked in part of the trials for failures to respond by the end of the CS-US interval.

As concerns the data from the reversal period, the differences in AA reacquisition between the $\mathrm{L}^{+} \mathrm{NL}^{-}$ group (formerly $\mathrm{N}^{+} \mathrm{LN}-$ ) and the $\mathrm{N}^{+} \mathrm{LN}^{-}$group (formerly $\mathrm{L}^{+} \mathrm{NL}^{-}$) may be ascribed to the different amount of inhibitory control exerted, respectively, by light and by noise at the end of the original training period. Both groups with compound nonreinforced stimuli showed little evidence of extinction during 16 reversal sessions. The most economic explanation of this phenomenon appears to be that the reversal of difficult discriminations with compound no-go stimuli creates a "ceiling" of high responding to these stimuli, leaving little room for the appearance of stimulus nonequivalences. Obviously, additional data on more extended reversal training are required, which may or may not show a reappearance of stimulus interactions at a later time.

A higher frequency of responses to nonreinforced noise than to nonreinforced light was found during the reversal of the $\mathrm{L}^{+} \mathrm{N}^{-}$and $\mathrm{N}^{+} \mathrm{L}^{-}$discriminations. Such stimulus nonequivalence extends the possible implications of the $\mathrm{L}^{+} \mathrm{NL}^{-}$vs $\mathrm{N}^{+} \mathrm{LN}^{-}$nonequivalence. In fact, this finding not only confirms that there is a general preparedness for light to act as suppressor of locomotion, but it also suggests that the relative inability of noise to act as suppressor depends on a "go" tendency determined either by the concurrent presentation of the actual AA signal or by the previous use of noise itself as AA signal $\left(\mathrm{L}^{+} \mathrm{N}^{-}\right.$training after $\mathrm{N}^{+} \mathrm{L}^{-}$ training). This conclusion is further strengthened by the results of an additional experiment, not to be reported in detail, showing that stimulus nonequivalences are maintained when using an identical no-go stimulus 
complex in the $\mathrm{L}^{+} \mathrm{NL}^{-}$and the $\mathrm{N}^{+} \mathrm{LN}^{-}$tasks (simultaneous light and noise onset and offset instead of a $1-\mathrm{sec}$ interval between onset of the first presented stimulus and onset of the AA signal).

The above findings and those reported in previous papers rule out that stimulus nonequivalences in go/no-go avoidance discriminations can be explained by a preparedness factor, specific for a signaling system that is responsible for motor act differentiation control under a given drive operation (Konorski, 1967; see also Introduction to Experiment II). Alternative, highly hypothetical explanations will be examined in the following section, since they require a discussion of stimulus and response factors in classical fear conditioning and inhibition, of possible mechanisms underlying preparedness phenomena in avoidance learning, and of interactions between response-dependent and response-independent stimulus events used to signal "danger" and "safety" in several different situations.

\section{GENERAL DISCUSSION}

The data reported and discussed above rule out simple explanations of the stimulus nonequivalences observed both in the acquisition of successive avoidance discriminations with a compound PA or extinction signal and in the reversal of discriminations with simple go and no-go signals. In fact, Experiment I excluded that the differences between the $\mathrm{L}^{+} \mathrm{NL}^{-}$and the $\mathrm{N}^{+} \mathrm{LN}^{-}$tasks are due to perceptual or attentional deficits occurring with particular combinations of a light and a noise stimulus. On the other hand, Experiment II showed stimulus nonequivalences in AA-extinction tasks similar to those found in AA-PA tasks. On the basis of previous considerations (Introduction and Discussion of Experiment II), this allows us to conclude that one is dealing with a fairly general element of preparedness, emerging as a high-order interaction between sensory and response factors, rather than with a more specific type of preparedness influencing mainly response choice under the same drive operation.

Given the above negative conclusions, only tentative working hypotheses can be proposed to explain the data and make testable predictions. One of these explanations assumes that the preparedness to use a given stimulus for the modulation of drive states is closely related to the preparedness to use the same stimulus for the activation or suppression of a particular response in a particular drive state. It is well known that both light and noise can easily acquire fear-enhancing or fear-inhibiting properties in classical conditioning tests. In some CER experiments by Kamin (1969), it has also been shown that response suppression can develop slightly more rapidly with a light than with a noise CS. At this point, more extended experiments on classical fear conditioning, differentiation, and inhibition, using different stimulus combinations and opposite types of response criteria, could test the hypothesis of related preparedness elements in drive and response modulation. For example, the hypothesis would be supported by the finding of stimulus nonequivalences like those observed in instrumental discriminations when using activation measures (e.g., when classical $\mathrm{CS}^{+}, \mathrm{CS}^{-}$, and $\mathrm{CI} / \mathrm{CS}^{+}$are superimposed on nondiscriminated two-way avoidance), while opposite results, or at least no stimulus nonequivalences, should be found with suppression measures (CER tests).

The above considerations should be viewed in connection with recent hypotheses on the existence of various categories of avoidance responses, requiring a greater or lesser reliance on conventional mediating processes as defined, e.g., by the two-factor theory. Bolles (1972) has emphasized a series of preparedness factors that are explained on the basis of the relationship between the avoidance response required and species-specific defensive responses (SSDRs). Anisman and Waller (1973) have extended this analysis by considering a series of experiential factors which favor the emergence of one or the other SSDR, particularly running vs freezing. Zielinski (1972) has followed an alternative approach by considering short-latency and long-latency subclasses within a given response class. His tentative conclusion, implying a close analogy between preparedness phenomena in avoidance and "autoshaping" phenomena in situations with positive reinforcement (see discussion in Jenkins \& Moore, 1973), is that short-latency avoidance responses "based on mechanisms in which the emotional state plays a little role [Zielinski, 1972, p. 412]" can be explained on the basis of classical conditioning phenomena. This suggests that, while leaving unaltered the AA paradigm with light vs noise as CS, one should investigate the effects of different contingencies between compound stimuli and reinforcement in the absence of a response-reinforcement contingency during the compound stimuli themselves (classical paradigm and "truly random" and explicitly unpaired controls). In case similar stimulus nonequivalences are maintained in this situation, and experiments carried out entirely according to the classical paradigm (see above) cannot account for such stimulus nonequivalences, one should conclude that preparedness models so far proposed by previous investigators and in the present paper are grossly oversimplified. The provisional conclusion would be, rather, that the preparedness (or contrapreparedness) to emit a given response to a given signal can be independent of response-reinforcement contingencies during the presentation of the signal itself but dependent on response-reinforcement contingencies during other signals presented in the same situation.

An additional point should be considered here in relationship to the above problems, since it widens the range of testable predictions that can be derived from tentative preparedness models. Stimulus nonequivalences of the $\mathrm{L}^{+} \mathrm{NL}^{-}$vs $\mathrm{N}^{+} \mathrm{LN}^{-}$type have been so far described in discrete trial two-way situations, which create conflict between a given SSDR (running) and an absence of 
positional cues for the signaling of danger and safety. In such situations, presence or absence of discrete stimulus events upon response completion can tip the balance in favor of one or the other of two high-priority SSDRs, i.e., running, which makes the animal an efficient avoider, and freezing, which leaves the animal with a high punishment rate (Bolles, 1970, 1972). Therefore, several possibilities must be considered concerning the above stimulus interactions. First, stimulus nonequivalences could be found in a wide variety of go/no-go avoidance discriminations with symmetrical and asymmetrical reinforcement, which would support the general validity of one or the other of the above preparedness models. Second, stimulus nonequivalences could appear only when a locomotor response is required as the AA response, independently of whether or not an exteroceptive feedback is necessary for efficient AA behavior (one-way and two-way avoidance, wheel running avoidance, circular avoidance). This would introduce an additional limiting factor, i.e., the models would apply only to activation and suppression of locomotor responses in the presence of various AA, PA, and extinction stimuli. Third and fourth, stimulus nonequivalences could be limited to situations requiring discrete, response-produced exteroceptive feedbacks for efficient avoidance learning, either with a variety of different responses (two-way, wheel turning, and leverpress avoidance) or only in the case of bidirectional locomotor responses.

Should reliance on an exteroceptive response feedback in AA trials be the critical factor for the appearance of stimulus nonequivalences, then the interactions between types of stimuli and types of response-reinforcement contingencies should be restated as interactions between response-independent and response-dependent stimulus events signaling danger and safety. For example, the relative unpreparedness to use an acoustic signal for drive and response inhibition (in the presence of the visual AA signal or when noise itself has previously been used as AA signal) could depend on a particular limiting factor, namely, the reliance on response-produced light offset as the safety signal conditioned in AA trials.

On the one hand, this would explain the differences, if any, between classical discriminations using activation measures, in which response-produced stimulus events do not have a role as safety signals, and go/no-go instrumental discriminations. On the other hand, the taking into account of exteroceptive response feedbacks could explain similar stimulus nonequivalences in avoidance discriminations with compound no-go stimuli and either asymmetrical or symmetrical reinforcement. In an extinction trial, the situation can be interpreted as safe when the first presented stimulus acquires fear-inhibiting properties sufficient to overcome the effects of the AA signal, independently of the animal's behavior. In a PA trial, the situation can be interpreted as safe only on the basis of a compound including the first presented signal and interoceptive feedbacks provided by "behavior other than the AA response." In either case, light appears to be more apt to signal safety in the presence of the noise AA signal than vice versa, at least when locomotion is the required $\mathrm{AA}$ response and CS termination is essential for efficient AA learning.

\section{REFERENCES}

Anisman, H., \& Waller, T. G. Effects of inescapable shock on subsequent avoidance performance: Role of response repertoire changes. Behavioral Biology, 1973, 9, 331-355.

Biederman, G. B. Discriminated avoidance conditioning: Stimulus function in shaping and training. Psychonomic Science, 1967, 9, 263-264.

Bolles, R. C. Species-specific defense reactions and avoidance learning. Psychological Review, 1970, 71, 32-48.

Bolles, R. C. The avoidance learning problem. In G. H. Bower (Ed.). The psychology of learning and motivation. New York: Academic Press, 1972.

Carro-Ciampi, G., \& Bignami, G. Effects of scopolamine on shuttle-box avoidance and go-no go discriminations: Response-stimulus relationships, pretreatment baselines, and repeated exposure to drug. Psychopharmacologia, 1968, 13, 89-105.

Dobrzecka, C., Szwejkowska, G., \& Konorski, J. Qualitative versus directional cues in two forms of differentiation. Science, 1966, 153, 87-89.

Frontali, M., \& Bignami, G. Go/no-go avoidance discriminations in rats with simple "go" and compound "no-go" signals: Stimulus modality and stimulus intensity. Animal Learning \& Behavior, 1973, 1, 21-24.

Ison, J. R., \& Hammond, G. R. Modification of the startle reflex in the rat by changes in the auditory and visual environments. Journal of Comparative \& Physiological Psychology, 1971, 75, 435-452.

Jenkins, H. M., \& Moore, B. R. The form of the autoshaped response with food or water reinforcers. Journal of the Experimental Analysis of Behavior, 1973, 20, 163-181.

Kamin, L. J. Predictability, surprise, attention and conditioning. In B. Campbell and $\mathrm{R}$. Church (Eds.), Punishment and aversive behavior. New York: Appleton-Century-Crofts, 1969.

Katzev, R. D., \& Enkema, S. J. Acquisition and extinction of signaled avoidance as a function of intermittent reinforcem ent. Learning \& Motivation, 1973, 4, 176-196.

Konorski, J. Integrative activity of the brain. Chicago: University of Chicago Press, 1967.

Eawicka, W. The role of stimulus modality in successive discrimination and differentiation learning. Bulletin de l'Academic Polonaise des Sciences. Série des Sciences Biologiques, 1964, 12, 35-38.

Mackintosh, N. J. An analysis of overshadowing and blocking. Quarterly Journal of Experimental Psychology, 1971, 23, 118-125.

Myers, A. K. Discriminated operant avoidance learning in Wistar and $G-4$ rats as a function of type of warning stimulus. Journal of Comparative \& Physiological Psychology, 1964, 58, 453-460.

Revusky, S., \& Garcia, J. Learned associations over long delays. In G. H. Bower (Ed.), The psychology of learning and motivation. New York: Academic Press, 1970.

Rosic, N., \& Bignami, G. Scopolamine effects on go-no go avoidance discriminations: Influence of stimulus factors and primacy of training. Psychopharmacologia, 1970, 17, 203-215.

Rosic, N., Frontali, M., \& Bignami, G. Stimulus factors affecting go-no go discrimination learning by rats. Communications in Behavioral Biology, 1969, 4, 151-156.

Seligman, M. E. P. On the generality of the laws of learning. Psychological Review, 1970, 77, 406-418.

Shettleworth, S. J. Constraints on learning. In D. S. Lehrm an, R. A. Hinde, and E. Shaw (Eds.), Advances in the study of behavior. New York: Academic Press, 1972.

Smith, O. A., Mc Farland, W. L., \& Taylor, E. Performance in a shock-avoidance situation interpreted as pseudoconditioning. Journal of Comparative \& Physiological Psychology, 1961, 54, 154-157.

Weiss, S. J. Stimulus compounding in free-operant and classical conditioning: A review and analysis. Psychological Bulletin, 1972, 78, 189-208.

Winer, B. J. Statistical principles in experimental design. New York: McGraw-Hill, 1962.

Zielinski, $K$. Effects of prefrontal lesions on avoidance and escape reflexes. Acta Neurobiologiae Experimentalis, 1972, 32, 393-415.

(Received for publication May 31, 1973; revision received March 9,1974 ; accepted March $11,1974$. 